\title{
Behaviour of bridges under spatially non-uniform earthquake motions
}

\author{
Nawawi Chouw \\ University of Auckland, New Zealand \\ n.chouw@auckland.ac.nz
}

\begin{abstract}
Because the soil development along the bridge is never uniform, the spreading seismic waves will be altered by the spatially unequal soil properties along the wave path. In addition, seismic waves have a limited speed. Seismic waves will therefore arrive at the supports of adjacent bridge pier at different instants. Consequently, the bridge will experience spatially non-uniform ground excitation. The research focuses on the consequence of the spatial variation of ground motions for the bridge response. The earthquake is simulated by three large-scale shake tables that excite the bridge structure and adjacent abutments separately. The behaviour of the bridge structure is discussed.
\end{abstract}

